Permutation symmetry in chemical and physical problems

Molecular Symmetry and Spectroscopy. By P.R. Bunker. Pp. 424. (Academic: New York, 1979.) \$33.

THIS volume is the first comprehensive treatment of the use of permutation symmetry in molecules, that is, in chemical and physical problems. As such, it will be widely welcomed, and fills a void which has long plagued those who had a need for this material. Fortunately, the writing in this volume is extremely lucid.

The volume begins with a clear definition of permutation operations and groups, then adds the (space) inversion operation to lead to the complete nuclear permutation inversion group (CNPI). The author next leads the reader through the more conventional application of group theory to molecules, with, however, a quite unconventional set of emphases. This reviewer feels that persons previously unfamiliar with chemical applications of group theory would have considerable difficulty with the treatment given. Fortunately, however, one may assume today (and it seems that the author has done so, although he claims the contrary) that anyone interested in the subject matter of this book has previously attained a working knowledge of molecular point groups and their use in spectroscopic problems. The emphasis in the appropriate chapters of this volume lies with the symmetry properties of the Hamiltonian and of its eigenfunctions, a much more fundamental approach than that com- monly pursued. After these preliminaries are dealt with in $\mathbf{8}$ chapters the real meat of the volume follows: the definition of the molecular symmetry group on the basis of permutation and space inversion and its applications, primarily to problems of molecular spectroscopy and to non-rigid molecules.

It is to be welcomed that the uurhor has been very careful and explicit in his definitions. Thus, the emphasis on the distinction between space and molecule fixed coordinate systems has led to a clean and unambiguous distinction of the two inversion operations, $E^{*}$ and $i$, which have been far from clear in much of the previous literature. Similarly the very careful phrasing of the conditions of feasibility, although they may at times seem redundant, make a significant contribution to the understanding of the subject matter.

The book is extensively illustrated with well-drawn figures, essential when discussing symmetry operations and their effect. The author has attempted, and it seems successfully, to illustrate each theoretical concept he introduces immediately with appropriate examples. For further emphasis, throughout the volume he has provided problems to illustrate the principles, and then given detailed solutions.

Although this book addresses itself to a relatively restricted audience spectroscopists, and quantum mechanicians interested in classificaion of excited states - it may be expected to rapidly become an essential tool to this audience. It is further to be hoped that the volume will help to make more scientists aware of permutation symmetry and thereby provide new insights into other phenomena.

H.H. Jaffe

H.H. Jaffe is Professor of Chemistry at the University of Cincinnati, Ohio.

\section{Electron microscopy in chemical investigations}

The Chemical Applications of Transmission Electron Microscopy. By J. R. Fryer. Pp.286. (Academic: London and New York, 1979.) £18; \$38.

THE electron microscope finds application in almost all branches of science and technology. Over the years there have appeared many books describing its uses in biology, medicine, metallurgy and materials science, but we have had to wait until now for a treatment of its role in chemical research. The reasons for such a lack are not far to seek. Chemistry is rarely as interested in morphology (except in polymers) as are biology and metallurgy for differing purposes. Its problems so often involve knowledge at the atomic and molecular level, whether of structure or function. In recent years, however, the instrument has found increasing application in surface chemistry (catalysts and thin films), crystal chemistry (defects, non-stoichiometry and phase changes), solution chemistry (clay minerals, polymers) and in chemical kinetics (solidstate reactions, intercalation).

Progress in these new fields has now been brought together and critically presented by an active worker. Description of the various applications is preceded by four chapters which set out the physical principles and operational techniques. The microscope itself is dealt with briefly, fuller accounts being readily available. Greater attention is paid to the interaction of electrons with the specimen, including radiation damage, and to the mechanism of image formation and contrast. A separate chapter is devoted to the special conditions of high resolution microscopy, where phase contrast and lattice imaging are principally involved. The design of ancillary apparatus, with particular reference to special stages, and the various methods of specimen preparation, are also surveyed.

The meat of the book, however, is comprised in the later four chapters describing the use of electron microscopy in a variety of chemical investigations. Not unnaturally, greatest space is accorded to catalysts and their reactions, to the structure of crystals (especially graphite) and to molecular crystals and radiation damage to them, all subjects in which the author has made important contributions. These chapters are very fully illustrated with micrographs, not always well reproduced in my copy of the book, and line diagrams. Polymers and proteins receive less attention here; they are adequately covered elsewhere in the literature. In spite of the blurb on the cover, there is very little on the use of the electron microscope in biochemistry and materials science, but after all Dr Fryer did not set out to write an encyclopaedia of its applications.

He has done very well within this restricted plan, and the book can be highly recommended to research chemists who wish to know what this comparatively new technique could do for them. Two reservations must be stated, however. First, the references are not so completely up-to-date as could be desired. Apart from publications from his own laboratory, there are only three or four post-1975 references. The consequent omission of a great deal of recent research, especially at high resolution, is much to be regretted and is probably ascribable to delays in printing and binding, which seem to be on the increase. My second reservation concerns the price. Little more than a decade ago we could expect a well produced scientific book to cost about $£ 1$ per 100 pages. The price of this volume works out at well over six times as much, with some sacrifice in quality. Though this may be of the same order as the increase in price of a motorcar, it outstrips the rise in chemists' salaries. Sadly, few of them will be able to afford Dr Fryer's excellent monograph, but I urge librarians in spite of equally restricted resources to get it for their shelves.

V. E. Cosslett

V. E. Cosslett is Emeritus Reader in Electron Physics in the Cavendish Laboratory, University of Cambridge, $U K$. 